\title{
A Case of Pre-Existing Pregnancy During an In-Vitro Fertilization Cycle
}

\author{
Poh Ting Lim ${ }^{a}$, Jerry Kok Yen Chan ${ }^{b}, \mathrm{c}$, Melissa D. Tharmalingam ${ }^{\mathrm{b}}$, Sadhana Nadarajah ${ }^{\mathrm{b}}$, \\ Veronique Viardot-Foucault ${ }^{\mathrm{b}}$
}

\begin{abstract}
Serum progesterone levels performed prior to oocyte pick-up is commonly used to guide embryo transfer in in-vitro fertilization (IVF) cycles, as elevated levels can negatively influence pregnancy outcomes. However, levels associated with normal pregnancies should trigger clinicians to consider alternative causes such as a pre-existing pregnancy. We report a case of a 37-year-old patient who underwent controlled ovarian hyperstimulation in a gonadotrophin-releasing hormone antagonist cycle while having an undetected early pregnancy. No oocytes were retrieved at oocyte retrieval despite adequate follicular responses. Her serum progesterone level on the day of her trigger injection was $57.8 \mathrm{nmol} / \mathrm{L}$. She was found to have a pregnancy of unknown location, detected 3 weeks after her oocyte retrieval and was subsequently treated with systemic methotrexate.
\end{abstract}

Keywords: Pregnancy; Progesterone; In-vitro fertilization; Superfetation

\section{Introduction}

During the follicular phase of the menstrual cycle, serum progesterone levels rise slightly in the late follicular phase with follicular growth in response to gonadotropin stimulation [1]. It then rises significantly following ovulation and peaks approximately a week later, remaining high should pregnancy occur, and falls to an undetectable level a couple of days before the occurrence of the periods in an unfertilized cycle [2]. In in-vitro fertilization (IVF) cycles, the rise in serum progesterone is amplified by

Manuscript submitted July 22, 2021, accepted August 13, 2021

Published online August 25, 2021

aDivision of Obstetrics and Gynecology, KK Women's and Children's Hospital, Singapore

bDepartment of Reproductive Medicine, KK Women's and Children's Hospital, Singapore

${ }^{\mathrm{c}}$ Corresponding Author: Jerry Kok Yen Chan, Department of Reproductive Medicine, KK Women's and Children's Hospital, 100 Bukit Timah Road, Singapore 229899, Singapore. Email: jerry.chan.k.y@singhealth.com.sg

doi: https://doi.org/10.14740/jmc3752 multiple follicular developments and high steroidogenesis [3]. Progesterone causes decidualization of the endometrium, converting it from a proliferative to a secretory phase, in preparation for implantation [3]. Premature progesterone elevations are shown to result in premature secretory transformation of the endometrium, reducing embryo-endometrium synchrony and thus impairing implantation [4]. It has become common practice to perform serum progesterone levels on the human chorionic gonadotropin (HCG) trigger day to determine if patients are suitable for a fresh embryo transfer, with cut-off value varying widely from 0.4 to $2 \mathrm{ng} / \mathrm{mL}(1.3-6.3 \mathrm{nmol} / \mathrm{L})$ [4].

Women undergoing IVF typically start their controlled ovarian hyperstimulation cycles on day 2 of their menstrual cycles to allow for recruitment of follicles and hence presumed to be non-pregnant. However, should the serum progesterone levels at any point of the IVF cycle be markedly elevated, particular beyond ovulatory levels, one should consider the possibility of an unsuspected pregnancy and a urine pregnancy test should be performed. We describe a case of an undetected pregnancy during IVF treatment at our center.

\section{Case Report}

\section{Investigations}

Our patient was a 37-year-old Indian lady who has been married for 10 years and trying to conceive for 18 months. She had no previous pregnancies and reported regular menstrual periods, ranging between 26 and 28 days. Her partner was a 37 -year-old man with no past medical history. Prior to presenting to our institution, she had undergone two cycles of clomiphene citrate-based ovulation induction and two cycles of nonstimulated intrauterine insemination unsuccessfully. Basal investigations at our center revealed a normal ovarian reserve (anti-Mullerian hormone level of $2 \mathrm{ng} / \mathrm{mL}$, antral follicle count of 14, a normal pelvic ultrasound and hysterosalpingogram). Her husband's semen analysis showed hypospermia $(1 \mathrm{~mL})$, normal motility $(47 \%)$ and counts (46 million/mL), and mild teratozoospermia $(3 \%)$.

The couple decided to proceed with IVF treatment and had their first gonadotropin-releasing hormone $(\mathrm{GnRH})$ antagonist cycle in November 2020. Her last menstrual period was on November 22, 2020. The following day, an ultrasound scan showed an endometrium thickness of $4 \mathrm{~mm}$ with small 


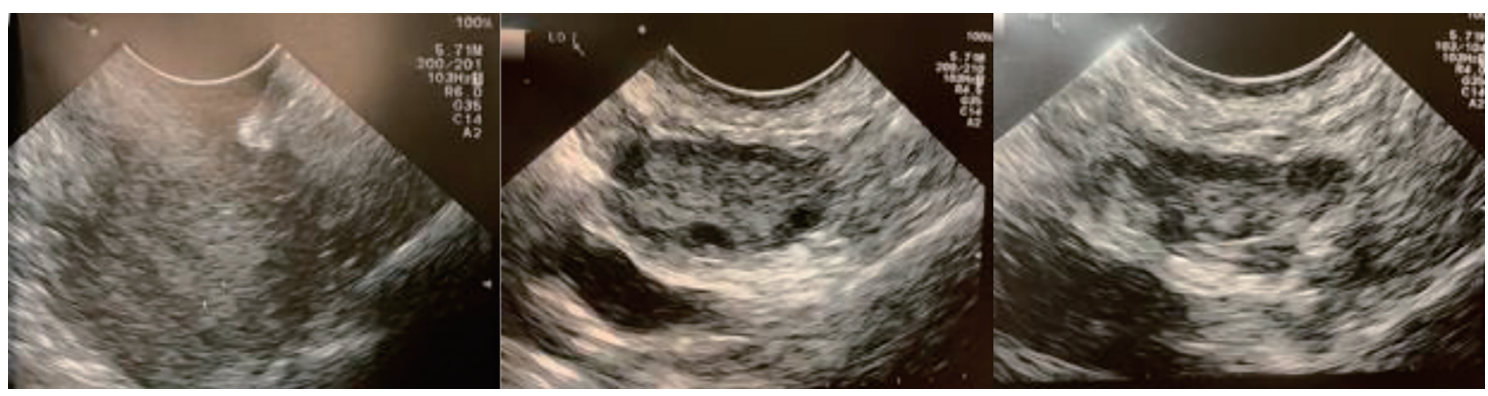

Figure 1. Ultrasound images on day 2 of stimulation cycle. Left: endometrium thickness; middle: left ovary; right: right ovary. Small antral follicles in both ovaries.

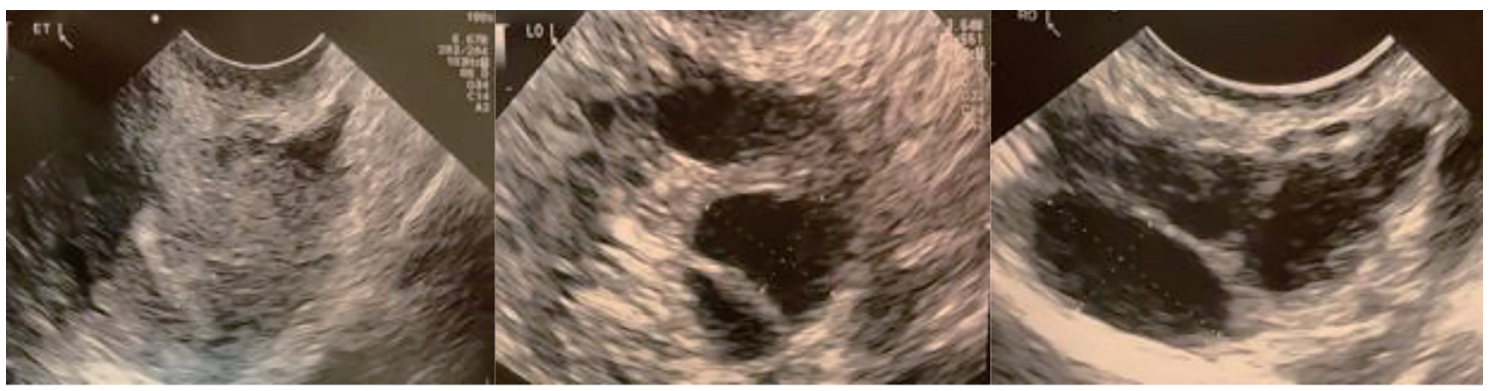

Figure 2. Ultrasound images on day 10 of stimulation cycle. Left: endometrium thickness; middle: left ovary; right: right ovary.

antral follicles in both ovaries (Fig. 1), and controlled ovarian hyperstimulation with recombinant human follicle-stimulating hormone (FSH, Gonal-F, Merck Serono SpA, Italy) at a dose of 225 IU daily was commenced. Subcutaneous GnRH antagonist ganirelix (Orgalutran, Vetter Pharma-Fertigung $\mathrm{GmbH} \&$ Co., Germany) $250 \mu \mathrm{g}$ was fixed started on the fifth day of stimulation. Follicular tracking scans performed periodically showed adequate growth of the follicles. On day 10 of simulation, she complained of per-vaginal spotting and the scan showed a $15 \mathrm{~mm}$ cyst of indeterminate nature on the left ovary (Fig. 2). An adequate follicular response was achieved by day 11 with four follicles larger than $17 \mathrm{~mm}$ and five follicles between 14 and $16 \mathrm{~mm}$ (Fig. 3). Final follicular maturation was achieved with $250 \mu \mathrm{g}$ of subcutaneous recombinant choriogonadotropin alfa (Ovidrel, Merck Serono S.p.A, Italy). A routine serum progesterone level performed on that day was $57.8 \mathrm{nmol} / \mathrm{L}(18.2 \mathrm{ng} / \mathrm{mL})$. Transvaginal oocyte retrieval was performed $36 \mathrm{~h}$ later, but aspiration of all the expected follicles in both ovaries only produced clear follicular fluid without any oocytes or cumulus cells seen.

\section{Diagnosis}

The patient was informed of the presence of empty follicles likely secondary to premature ovulation, with a differential diagnosis of empty follicle syndrome. This led to the cancellation of the cycle with anticipated period 10 - 14 days later. As she remained amenorrheic, she performed a urine pregnancy test on December 26, 2020 which yielded a positive result. The pregnancy was confirmed on a serum beta-HCG test on January 5, 2021, showing a level of 3,829 $\mathrm{mIU} / \mathrm{mL}$. She denied having had any sexual intercourse since her last menstrual period prior to starting the antagonist cycle.

A repeat serum beta-HCG test on January 7, 2021 was $3,105 \mathrm{mIU} / \mathrm{mL}$, compatible with a sub-optimal trend. She did not report any abdominal pain or per-vaginal bleeding. A diagnosis of a pregnancy of unknown location was made following

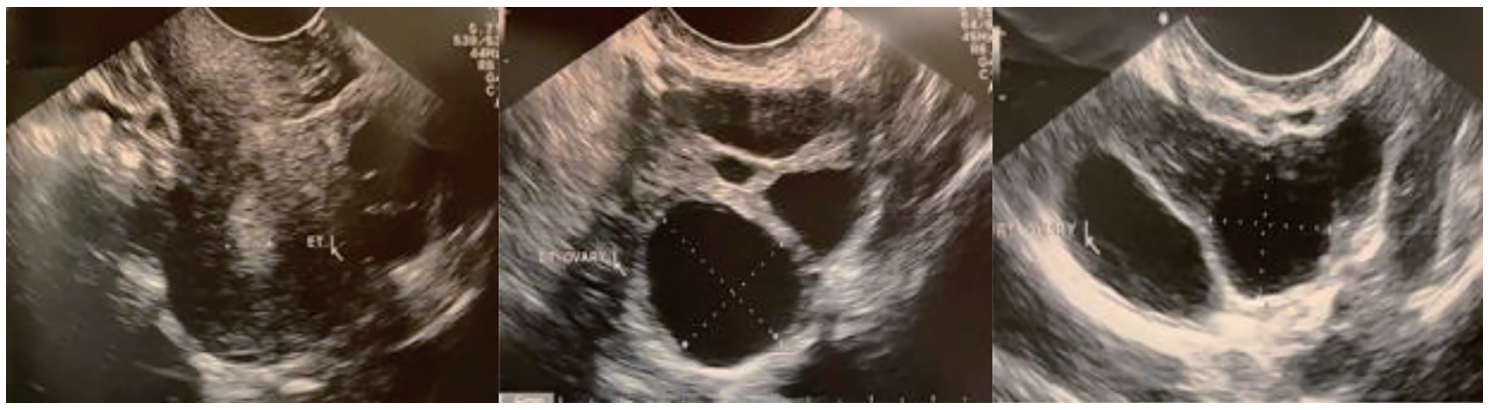

Figure 3. Ultrasound images on day 11 of stimulation cycle. Left: endometrium thickness; middle: left ovary; right: right ovary. 


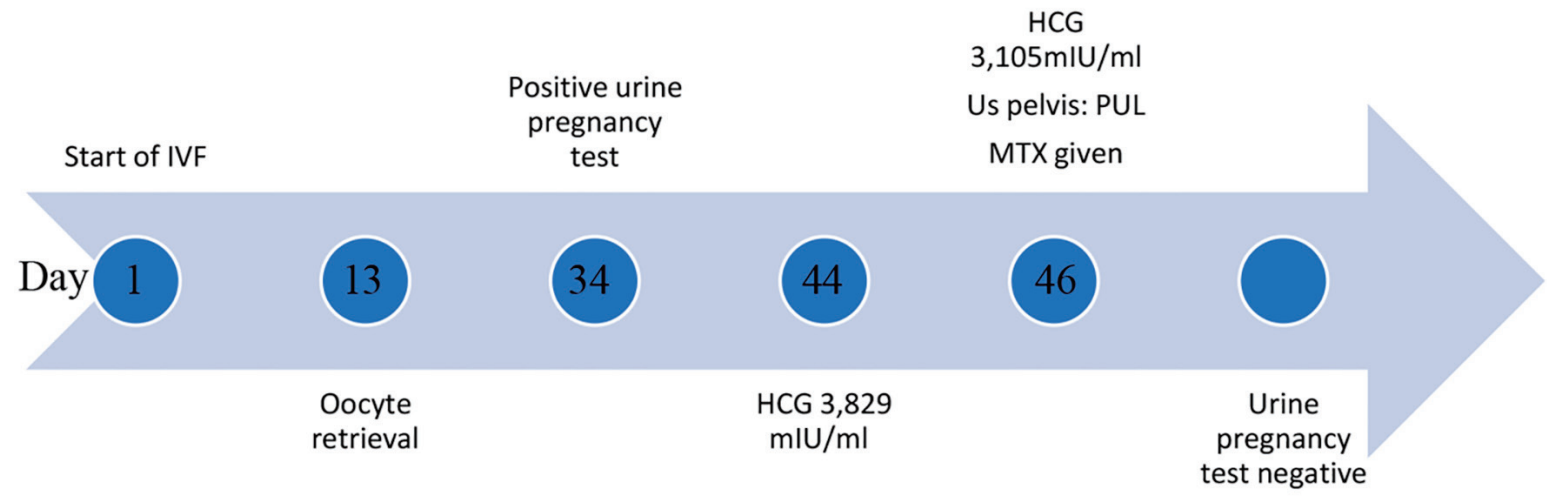

Figure 4. Timeline of events.

a transvaginal ultrasound of the pelvis which showed an empty uterus. Multiple corpus luteal cysts were present in both ovaries and there were no adnexal masses or free fluid seen.

\section{Treatment}

The failing pregnancy of unknown location was successfully treated with intramuscular dose of methotrexate based on her body surface area.

\section{Follow-up and outcomes}

The serum beta-HCG levels and urine pregnancy test were both negative a month later (Fig. 4).

\section{Discussion}

We presented above the case of an undetected pregnancy in a patient prior to starting her IVF cycle. The ultrasound performed on the second day of her periods was normal and her controlled ovarian stimulation was unremarkable except for the presence of a left ovarian cyst and an unusually high progesterone level on the day of trigger.

While patients undergoing IVF are deemed subfertile, a Cochrane review comparing IVF with expectant management in women with unexplained infertility revealed that $1 \%$ of women achieved spontaneous conception following expectant management for up to an average of 4 years [5]. Troude et al also studied 2,134 couples who underwent IVF and found that $24 \%$ of the couples achieved spontaneous pregnancies despite having failed IVF previously [6].

Even though our patient was started on her ovarian stimulation on day 2 of her "regular menstrual flow", it is likely that she was already pregnant, with implantation bleeding mistaken as menstrual flow. It is a rare occurrence, however, for women to achieve ovulation during a pre-existing pregnancy. In a phenomenon known as superfetation, commonly seen in other animal species, ovulation, fertilization and embryo implantation occur during an ongoing pregnancy, resulting in a pregnancy with fetuses of different gestational ages [7]. This rarely happens in the human species due to the pituitary suppression of gonadotrophins from the high levels of estradiol and progesterone in pregnancy. However, this suppression can be overcome by the administration of exogenous gonadotrophins, stimulating follicular growth and eventually, ovulation.

In 1985, Serafini et al reported successful stimulation of folliculogenesis in a woman with an undiagnosed ectopic pregnancy, with successful retrieval and fertilization of the oocytes following laparoscopic salpingostomy [8]. In 2010, Lantieri et al reported a case of a 32-year-old woman who underwent ovarian stimulation with intrauterine insemination (IUI) with recombinant FSH [9]. She had four consecutive cycles of IUI and developed severe pelvic pain after her fourth cycle. She was diagnosed with a left tubal ectopic pregnancy of 6 weeks gestation, likely conceived through her third IUI cycle. She then underwent a laparoscopic salpingectomy and was found to have rising HCG levels post-operatively. An interval scan revealed a new development of a viable embryo in the uterus. This pregnancy progressed normally and she delivered a healthy baby at 39 weeks.

We speculate that our patient was already pregnant prior to the start of ovulation stimulation. The thin endometrium on the first day of ovarian stimulation could be a result of a possible ectopic pregnancy and the indeterminate cyst on the left ovary on day 10 of ovarian stimulation could have been a corpus luteum cyst. It is likely that exogenous gonadotrophins stimulated follicular growth despite the pregnant state and has allowed for follicular growth and ovulation. A differential diagnosis of empty follicular syndrome could also explain the failure to retrieve oocytes from mature ovarian follicles despite normal follicular development. This has an incidence of $0.045-3.5 \%$. Proposed etiologies include dysfunctional folliculogenesis, abnormal oocyte development and maturation, or premature ovulation [10].

It is a common practice to check serum progesterone prior to oocyte retrieval as an elevated serum progesterone on HCG trigger day is believed to negatively influence pregnancy outcome [4]. Causes of progesterone elevations include increased production from developing follicles, decreased progesterone metabolism to androgens and increased sensitivity to luteinizing hormone (LH) receptors [11]. Clinicians often resort to a segmented freeze-all technique for women with high serum 
progesterone levels and deemed unsuitable for fresh embryo transfer. However, cut-off values for progesterone level vary widely from 0.4 to $2 \mathrm{ng} / \mathrm{mL}$ (1.3 to about $6.3 \mathrm{nmol} / \mathrm{L})$ [4]. Serum progesterone levels ranges from 10 to $54 \mathrm{ng} / \mathrm{mL}(31.8$ - $171.7 \mathrm{nmol} / \mathrm{L})$ in mid-luteal phase and normal early pregnancy, and between 3 and $12 \mathrm{ng} / \mathrm{mL}(9.5-38.2 \mathrm{nmol} / \mathrm{L})$ in failing pregnancies [12]. An abnormally high progesterone level should raise suspicion of alternative causes, such as adrenal gland production, ovarian tumors, and imperatively, to rule out a case of a pre-existing pregnancy.

Lantieri et al proposed performing serum HCG measurement prior to the start of ovarian stimulation even if apparently normal menstruation has occurred [9], as implantation bleeding can occur in approximately $8 \%$ of women 1 month after conception [13]. While this would avoid missing a pre-existing pregnancy during IVF, superfetation is a rare occurrence and it is difficult to justify universal serum HCG testing prior to commencement of IVF cycles due to its cost and invasiveness. A less invasive option of performing a urine pregnancy test prior to the start of ovarian stimulation may be considered in patients with irregular menstrual periods or for those who report abnormal bleeding patterns prior to starting IVF. In addition, protocols should be in place to review the results of serum progesterone levels performed prior to HCG trigger so that patients with abnormally elevated progesterone can be recalled in time for a serum HCG test to exclude pregnancy before oocyte retrieval procedures.

\section{Acknowledgments}

None to declare.

\section{Financial Disclosure}

This report received no specific grant from any funding agency in the public, commercial, or not-for-profit sectors.

\section{Conflict of Interest}

The authors have no conflict of interest to disclose.

\section{Informed Consent}

Written informed consent for publication of their clinical details and/or clinical images was obtained from the patient.

\section{Author Contributions}

P.T. Lim is the main writer of this manuscript. J.K.Y. Chan is the corresponding author who conceptualized and supervised the writing of this manuscript. M.D. Tharmalingam is the embryologist who analyzed the fluid obtained during oocyte retrieval. V. Viardot-Foucault supervised and edited the manuscript. S. Nadarajah is the specialist in reproductive medicine involved in the care of this patient during her clinical visits.

\section{Data Availability}

The authors declare that data supporting the findings of this study are available within the article.

\section{References}

1. Couzinet B, Brailly S, Bouchard P, Schaison G. Progesterone stimulates luteinizing hormone secretion by acting directly on the pituitary. J Clin Endocrinol Metab. 1992;74(2):374-378.

2. Stricker R, Eberhart R, Chevailler MC, Quinn FA, Bischof P, Stricker R. Establishment of detailed reference values for luteinizing hormone, follicle stimulating hormone, estradiol, and progesterone during different phases of the menstrual cycle on the Abbott ARCHITECT analyzer. Clin Chem Lab Med. 2006;44(7):883-887.

3. Luesley DM, Kilby MD. Reproductive Gynaecology. In: Obstetrics and Gynaecology, an evidence-based text for the MRCOG, 3rd ed. CRC Press. 2016. p. 545-547.

4. Ashmita J, Vikas S, Swati G. The impact of progesterone level on day of hCG injection in IVF cycles on clinical pregnancy rate. J Hum Reprod Sci. 2017;10(4):265-270.

5. Kamphuis EI, Bhattacharya S, van der Veen F, Mol BW, Templeton A, Evidence Based IVFG. Are we overusing IVF? BMJ. 2014;348:g252.

6. Troude P, Bailly E, Guibert J, Bouyer J, de la Rochebrochard E, DAIFI Group. Spontaneous pregnancies among couples previously treated by in vitro fertilization. Fertil Steril. 2012;98(1):63-68.

7. Roellig K, Menzies BR, Hildebrandt TB, Goeritz F. The concept of superfetation: a critical review on a 'myth' in mammalian reproduction. Biol Rev Camb Philos Soc. 2011;86(1):77-95.

8. Serafini P, Yee B, Vargyas J, Marrs RP. Development of multiple ovarian follicles for in vitro fertilization in a patient with an undiagnosed ectopic pregnancy. Fertil Steril. 1985;43(4):656-658.

9. Lantieri T, Revelli A, Gaglioti P, Menato G, Gennarelli G, Delle Piane L, Massobrio M. Superfetation after ovulation induction and intrauterine insemination performed during an unknown ectopic pregnancy. Reprod Biomed Online. 2010;20(5):664-666.

10. Stevenson TL, Lashen H. Empty follicle syndrome: the reality of a controversial syndrome, a systematic review. Fertil Steril. 2008;90(3):691-698.

11. Kaponis A, Chronopoulou E, Decavalas G. The curious case of premature luteinization. J Assist Reprod Genet. 2018;35(10):1723-1740.

12. Radwanska E, Frankenberg J, Allen EI. Plasma progesterone levels in normal and abnormal early human pregnancy. Fertil Steril. 1978;30(4):398-402.

13. Speert H, Guttmacher AF. Frequency and significance of bleeding in early pregnancy. J Am Med Assoc. 1954;155(8):712-715. 